\title{
How to avoid unnatural hierarchical thermal leptogenesis
}

\author{
Jackson D. Clarke ${ }^{* \dagger}$ \\ ARC Centre of Excellence for Particle Physics at the Terascale, \\ School of Physics, University of Melbourne, 3010, Australia \\ E-mail: j.clarke5epgrad.unimelb.edu.au
}

\begin{abstract}
A one-flavour naturalness argument suggests that the Type I seesaw model cannot naturally explain neutrino masses and the baryon asymmetry of the Universe via hierarchical thermal leptogenesis. We prove that there is no way to avoid this conclusion in a minimal three-flavour setup. We then comment on the simplest ways out. In particular, we focus on a resolution utilising a second Higgs doublet. Such models predict an automatically SM-like Higgs boson, (maximally) $\mathrm{TeV}$-scale scalar states, and low- to intermediate-scale hierarchical leptogenesis with $10^{3} \mathrm{GeV} \lesssim M_{N_{1}} \lesssim 10^{7} \mathrm{GeV}$.
\end{abstract}

18th International Conference From the Planck Scale to the Electroweak Scale 25-29 May 2015

Ioannina, Greece

\footnotetext{
* Speaker.

${ }^{\dagger}$ Based on work completed in collaboration with Robert Foot and Raymond R. Volkas [1, 2].
} 


\section{Introduction}

The standard model (SM) and the paradigm of electroweak symmetry breaking realised by the Higgs potential $V_{\mathrm{SM}}=\mu^{2} \Phi^{\dagger} \Phi+\lambda\left(\Phi^{\dagger} \Phi\right)^{2}$, with $\mu^{2}\left(m_{Z}\right) \approx-(88 \mathrm{GeV})^{2}$, has been extremely successful in explaining low energy phenomena. However it fails to explain neutrino masses and the baryon asymmetry of the Universe (BAU). A straightforward way to explain both is to add three heavy right-handed neutrinos. Gauge invariance then allows two additional renormalisable terms in the Yukawa Lagrangian,

$$
-\Delta \mathscr{L}_{Y}=\left(y_{v}\right)_{i j} \overline{l_{L}^{i}} \tilde{\Phi} v_{R}^{j}+\frac{1}{2} M_{i} \overline{\left(v_{R}^{i}\right)^{c}} v_{R}^{i}+\text { h.c. },
$$

where $l_{L}=\left(v_{L}, e_{L}\right)^{T}, \tilde{\Phi}=i \tau_{2} \Phi^{*}$, and $M_{i}$ are the right-handed neutrino masses. This is the Type I seesaw model $[3,4,5,6]$. After $\Phi$ gains a vacuum expectation value (vev) $\langle\Phi\rangle=v / \sqrt{2} \approx 174 \mathrm{GeV}$, and if $y_{v} v \ll M_{i}$, the neutrino mass matrix is given by the seesaw formula

$$
m_{v}=\frac{v^{2}}{2} y_{v} \mathscr{D}_{M}^{-1} y_{v}^{T}
$$

where $\mathscr{D}_{M} \equiv \operatorname{diag}\left(M_{1}, M_{2}, M_{3}\right)$. The BAU can be produced via hierarchical thermal leptogenesis [7]: the $C P$ violating out-of-equilibrium decays of the lightest right-handed neutrino $N_{1}$ create a lepton asymmetry which is transferred to the baryon sector by electroweak sphalerons. The Davidson-Ibarra bound [8, 9] (ensuring enough $C P$ violation) for successful hierarchical $\left(M_{N_{1}} \ll\right.$ $M_{N_{2}} \ll M_{N_{3}}$ ) thermal leptogenesis is

$$
M_{N_{1}} \gtrsim 5 \times 10^{8} \mathrm{GeV}\left(\frac{v}{246 \mathrm{GeV}}\right)^{2},
$$

where $v$ is the vev that enters the seesaw Eq. 1.2. This appears to be in conflict with the naturalness argument for right-handed neutrinos made by Vissani [10]. In a one-flavour model, Vissani found (where $\mu_{R}$ is the renormalisation scale)

$$
\left|\frac{d \mu^{2}}{d \ln \mu_{R}}\right|=\left|-\frac{1}{4 \pi^{2}} y_{v} M_{N}^{2} y_{v}^{*}\right|<1 \mathrm{TeV}^{2} \quad \Rightarrow \quad M_{N} \lesssim 3 \times 10^{7} \mathrm{GeV}\left(\frac{v}{246 \mathrm{GeV}}\right)^{\frac{2}{3}}
$$

for a neutrino mass of $m_{v}=\frac{v^{2}}{2} \frac{y_{v}^{2}}{M_{N}} \approx 0.05 \mathrm{eV}$.

This proceedings paper addresses the following questions: can three-flavour effects ameliorate this conflict? and; if not, what can? In Sec. 2 the first question is answered in the negative. We outline our three-flavour treatment [1] which generalises the Vissani result to obtain three naturalness bounds:

$$
M_{N_{1}} \lesssim 4 \times 10^{7} \mathrm{GeV}, \quad M_{N_{2}} \lesssim 7 \times 10^{7} \mathrm{GeV}, \quad M_{N_{3}} \lesssim 3 \times 10^{7} \mathrm{GeV}\left(\frac{0.05 \mathrm{eV}}{m_{\min }}\right)^{\frac{1}{3}},
$$

where $m_{\min }$ is the lightest neutrino mass. These results confirm that natural $N_{1^{-}}, N_{2^{-}}$, or $N_{3^{-}}$ dominated hierarchical thermal leptogenesis is not possible in a minimal three-flavour Type I seesaw. In Sec. 3 we suggest some simple variations/extensions which reopen the possibility of a natural BAU. We focus on a two-Higgs-doublet solution recently proposed in our Ref. [2], motivated by the following observation: if $v \lesssim 30 \mathrm{GeV}$ in Eq. 1.2, then Eqs. 1.3 and 1.4 become compatible. We find viable natural models which predict a SM-like Higgs boson, (maximally) TeV-scale scalar states, and low- to intermediate-scale hierarchical leptogenesis with $10^{3} \mathrm{GeV} \lesssim M_{N_{1}} \lesssim 10^{8} \mathrm{GeV}$. 


\section{Electroweak naturalness in three-flavour Type I seesaw}

\subsection{Measurable naturalness}

After renormalisation, the physical effects of any heavy degree of freedom are embodied in the renormalisation group equations (RGEs). The RGEs are therefore a sensible way to quantify a physical and measurable (at least in principal) electroweak naturalness problem. Roughly, a problem arises whenever $d \mu^{2} / d \ln \mu_{R} \gtrsim \mu^{2}$; in such a case, $\mu^{2}\left(\mu_{R}\right)$ will evolve to large values, which one can interpret as a fine-tuning of $\mu^{2}$ at a high scale. Intuitive naturalness criteria are then: bound the RGE itself, or; quantify and bound the fine-tuning in the mass parameter evolved to some high scale $\Lambda_{h}$. That is:

$$
\left|\frac{1}{\mu^{2}\left(m_{Z}\right)} \frac{d \mu^{2}}{d \ln \mu_{R}}\right|<\mathscr{O}(1), \quad \text { or; } \quad \Delta\left(\Lambda_{h}\right)=\left|\frac{\mu^{2}\left(\Lambda_{h}\right)}{\mu^{2}\left(m_{Z}\right)} \frac{\partial \mu^{2}\left(m_{Z}\right)}{\partial \mu^{2}\left(\Lambda_{h}\right)}\right|<\mathscr{O}(1),
$$

where $\Delta$ is a Barbieri-Giudice style fine-tuning measure [11, 12]. Such criteria should not be taken too seriously (and nature may just be fine-tuned after all), but they can certainly serve as guiding principles which capture our subjective sense of physical naturalness (of mass parameters), and they are calculable in any perturbative model.

\subsection{Three-flavour seesaw}

Let us now examine right-handed neutrino corrections to the electroweak $\mu^{2}$ parameter in the three-flavour Type I seesaw model. We will invoke the Casas-Ibarra parameterisation $U y_{v}=$ $\frac{\sqrt{2}}{v} \mathscr{D}_{m}^{\frac{1}{2}} R \mathscr{D}_{M}^{\frac{1}{2}}$, where $R$ is an arbitrary unitary matrix, and $\mathscr{D}_{m} \equiv \operatorname{diag}\left(m_{1}, m_{2}, m_{3}\right)=U m_{v} U^{T}$ is the diagonalised neutrino mass matrix. The RGE for $\mu^{2}$ is

$$
\frac{d \mu^{2}}{d \ln \mu_{R}}=\frac{1}{(4 \pi)^{2}}\left[-4 \operatorname{Tr}\left[y_{v} \mathscr{D}_{M}^{2} y_{v}^{\dagger}\right]+\mathscr{O}\left(\mu^{2}\right)\right]=\frac{1}{(4 \pi)^{2}}\left[-4 \frac{2}{v^{2}} \operatorname{Tr}\left[\mathscr{D}_{m} R \mathscr{D}_{M}^{3} R^{\dagger}\right]+\mathscr{O}\left(\mu^{2}\right)\right] .
$$

Bounding directly each right-handed neutrino contribution by $1 \mathrm{TeV}^{2}$ (akin to Vissani) results in three bounds:

$$
M_{j} \lesssim 3 \times 10^{7} \mathrm{GeV}\left(\frac{v}{246 \mathrm{GeV}}\right)^{\frac{2}{3}}\left(\frac{0.05 \mathrm{eV}}{\sum_{i} m_{i}\left|R_{i j}\right|^{2}}\right)^{\frac{1}{3}},
$$

where $R_{i j}$ are the entries of $R$. We can always order the bounds by their size; we will call them $B_{j}$ and take $B_{1} \leq B_{2} \leq B_{3}$. The question we are interested in is: what values of $B_{j}$ are attainable from Eq. 2.3? To answer this question we need only extremise the $B_{j}$ over $R$. After a suitable parameterisation of $R$ and a numerical study we present our result for real $R$ in Fig. 1, as a function of the lightest neutrino mass in normal ordering $\left(m_{1}<m_{2}<m_{3}\right)$ and inverted ordering $\left(m_{3}<m_{1}<\right.$ $m_{2}$ ) scenarios. The result for complex $R$ with $\left|R_{i j}\right|<1$ (to avoid a fine-tuning) is similar. One can now plainly observe the generic naturalness bounds already written in Eq. 1.5.

What are the implications for leptogenesis? The Davidson-Ibarra bound (Eq. 1.3) for $N_{1^{-}}$dominated thermal hierarchical leptogenesis remains inconsistent with naturalness. An $\mathrm{N}_{2}$-dominated scenario is also inconsistent $[13,14,15]$. Lastly, it turns out that the same decoupling limit which allows $N_{3}$ to become naturally heavy also sends the $C P$ asymmetry in its decays to zero, excluding the possibility of a natural $N_{3}$-dominated scenario. Thus our results confirm that no minimal threeflavour Type I seesaw model can explain the neutrino masses and baryogenesis via hierarchical thermal leptogenesis while remaining completely natural. 

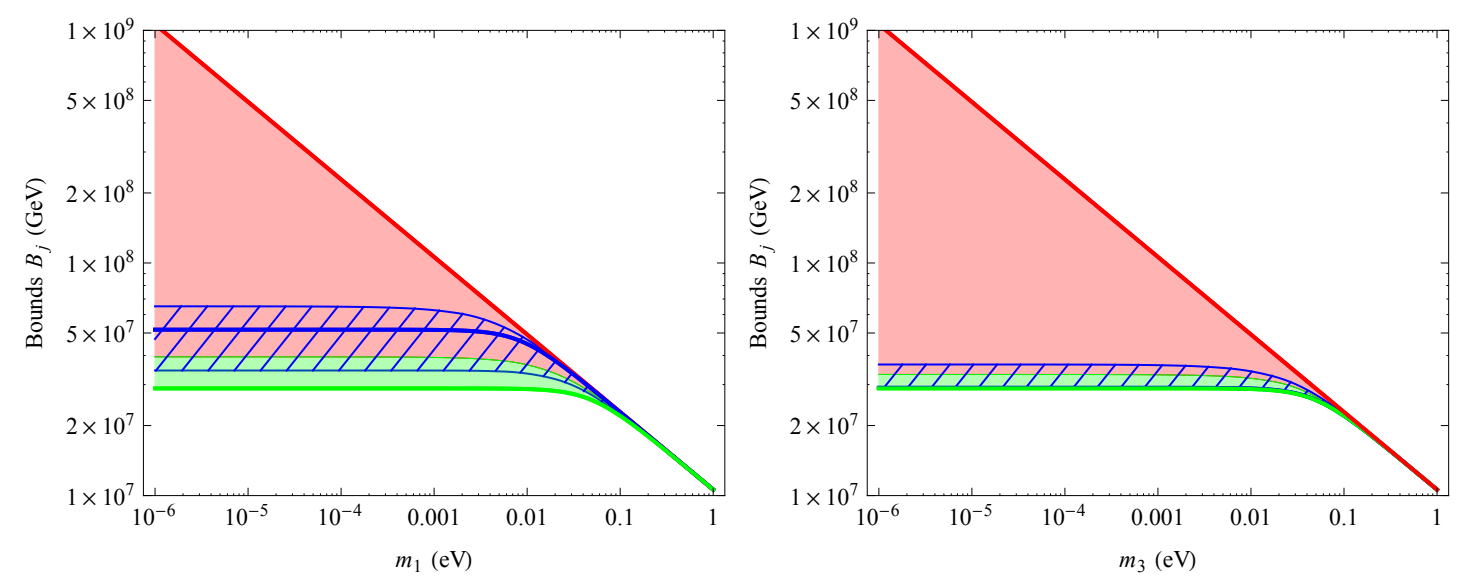

Figure 1: Left: As a function of the lightest neutrino mass in normal ordering, shown as red/bluehatched/green (darker/hatched/lighter) are the attainable values for $B_{3} \geq B_{2} \geq B_{1}$ naturalness bounds on the $M_{N_{i}}$, by requiring $d \mu^{2} / d \ln \mu_{R}<1 \mathrm{TeV}^{2}$. The regions assume $R$ is real. Thick solid lines show the case $R=\mathbb{I}$ (the complex $R$ case is similar). Right: As in Left but for inverted ordering. Note that the thick blue line is obscured by the thick green line. (Figure reproduced from Ref. [1]).

\section{Natural leptogenesis and neutrino masses with two Higgs doublets: the $v 2 \mathrm{HDM}$}

\subsection{How to avoid unnatural hierarchical thermal leptogenesis}

An obvious question is: in what minimal ways can we adapt the Type I seesaw to realise a natural BAU? There are a number of conspicuous possibilities: (1) lowering the Davidson-Ibarra bound, by considering dominant initial $N_{1}$ abundancy [9] ${ }^{1}$, resonant leptogenesis [16], a different baryogenesis mechanism entirely (such as neutrino oscillations [17]), or by introducing new fields which allow increased $C P$ violation in $N_{1}$ decays; (2) raising the naturalness bound by partially cancelling right-handed neutrino corrections $[18,19]$, or removing it entirely by restoring low-scale supersymmetry; (3) lowering the (possibly effective) vev entering the seesaw Eq. 1.2 so that the bounds of Eqs. 1.3 and 1.4 become consistent $(v \lesssim 30 \mathrm{GeV})$. Recently in Ref. [2] we implemented the latter possibility within a two-Higgs-doublet model with right-handed neutrinos ( $v 2 \mathrm{HDM})$. The remainder of this Section is dedicated to describing such models.

\subsection{The $v 2 \mathrm{HDM}$}

There are two doublets $\Phi_{1,2}$ with hypercharge +1 , and each gains a non-zero vev $\left\langle\Phi_{i}\right\rangle=$ $\left(0, v_{i} / \sqrt{2}\right)^{T}$ with $\sqrt{v_{1}^{2}+v_{2}^{2}}=v \approx 246 \mathrm{GeV}$ and $\tan \beta \equiv v_{1} / v_{2}$. As already motivated, we would like the vev contributing to the seesaw to be small. We therefore consider $v_{2} \ll v_{1}(\tan \beta \gg 1)$ and the following Yukawa Lagrangian:

$$
-\mathscr{L}_{Y}=+y_{u} \overline{q_{L}} \tilde{\Phi}_{1} u_{R}+y_{d} \overline{q_{L}} \Phi_{I} d_{R}+y_{e} \overline{l_{L}} \Phi_{J} e_{R}+y_{v} \bar{l}_{L} \tilde{\Phi}_{2} v_{R}+\frac{1}{2} M_{N} \overline{\left(v_{R}\right)^{c}} v_{R}+\text { h.c. },
$$

where $I, J$ define the $v 2$ HDM Type, and family indices are implied. The model Types are defined in Table 1. Note that, for Type II, LS, and Flipped arrangements, $y_{b, \tau}$ cause early Landau poles when $v_{2} \lesssim 4 \mathrm{GeV}(\tan \beta \gtrsim 70)$.

\footnotetext{
${ }^{1}$ The bound becomes $M_{N_{1}} \gtrsim 2 \times 10^{7} \mathrm{GeV}$, marginally consistent with the naturalness bound in Eq. 1.5.
} 


\begin{tabular}{c|c|c|c|c} 
Model & $u_{R}^{i}$ & $d_{R}^{i}$ & $e_{R}^{i}$ & $v_{R}^{i}$ \\
\hline Type I & $\Phi_{1}$ & $\Phi_{1}$ & $\Phi_{1}$ & $\Phi_{2}$ \\
Type II & $\Phi_{1}$ & $\Phi_{2}$ & $\Phi_{2}$ & $\Phi_{2}$ \\
Lepton-specific (LS) & $\Phi_{1}$ & $\Phi_{1}$ & $\Phi_{2}$ & $\Phi_{2}$ \\
Flipped & $\Phi_{1}$ & $\Phi_{2}$ & $\Phi_{1}$ & $\Phi_{2}$
\end{tabular}

Table 1: The $v 2$ HDM Types.

In order to construct a model with naturally small $v_{2}$ and potentially TeV-scale scalars, we softly break a symmetry which would otherwise imply $v_{2}=0$. For example, take the softly broken $U(1)$ symmetric potential

$$
\begin{aligned}
V_{2 \mathrm{HDM}}= & m_{11}^{2} \Phi_{1}^{\dagger} \Phi_{1}+m_{22}^{2} \Phi_{2}^{\dagger} \Phi_{2}-m_{12}^{2}\left(\Phi_{1}^{\dagger} \Phi_{2}+\Phi_{2}^{\dagger} \Phi_{1}\right) \\
& +\frac{\lambda_{1}}{2}\left(\Phi_{1}^{\dagger} \Phi_{1}\right)^{2}+\frac{\lambda_{2}}{2}\left(\Phi_{2}^{\dagger} \Phi_{2}\right)^{2}+\lambda_{3}\left(\Phi_{1}^{\dagger} \Phi_{1}\right)\left(\Phi_{2}^{\dagger} \Phi_{2}\right)+\lambda_{4}\left(\Phi_{1}^{\dagger} \Phi_{2}\right)\left(\Phi_{2}^{\dagger} \Phi_{1}\right),
\end{aligned}
$$

with $m_{11}^{2}<0, m_{22}^{2}>0$, and $m_{12}^{2} / m_{22}^{2} \ll 1$. In this case

$$
v_{2} \approx \frac{1}{1+\frac{v_{1}^{2}}{2 m_{22}^{2}}\left(\lambda_{3}+\lambda_{4}\right)} \frac{m_{12}^{2}}{m_{22}^{2}} v_{1}, \quad v_{1}=\sqrt{\frac{2}{\lambda_{1}}\left[\frac{1}{\tan ^{2} \beta}\left(m_{22}^{2}+\frac{1}{2} \lambda_{2} v_{2}^{2}\right)-m_{11}^{2}\right]} .
$$

In the limit $m_{22}^{2} \gg v_{1}^{2}\left(\lambda_{3}+\lambda_{4}\right), \lambda_{2} v_{2}^{2}$, we have $\tan \beta \approx m_{22}^{2} / m_{12}^{2}$ and $v_{1} \approx \sqrt{\frac{2}{\lambda_{1}}\left(-m_{11}^{2}+m_{12}^{2}\right)}$. This implies a relevant consistency condition, $2 m_{12}^{2} \lesssim \lambda_{1} v_{1}^{2}$, to ensure $m_{11}^{2}<0$ and avoid a fine-tuning for $v$. Typically we have $m_{12}^{2} \ll\left|m_{11}^{2}\right|$ so that $m_{11}^{2}$ sets the mass of the Higgs (as does $\mu^{2}$ in the SM).

The lightest $C P$ even boson $h$ obtains a mass $m_{h}^{2} \approx \lambda_{1} v_{1}^{2}$. Because of the approximate $U(1)$ symmetry, a notable side effect is an automatically SM-like $h$, i.e., there is no fine-tuning in the mixings to reproduce observations. ${ }^{2}$ The three extra scalar states $\left(H, A, H^{ \pm}\right)$have masses $\approx m_{22}$. Important constraints on $m_{22}$ in a given $v 2 \mathrm{HDM}$ Type are largely identical to those for a $2 \mathrm{HDM}$ of the same Type. These are: the consistency condition already mentioned; $m_{H^{ \pm}} \gtrsim 80 \mathrm{GeV}$ from direct searches at LEP [20]; $m_{22} \gtrsim 480 \mathrm{GeV}$ (for Type II and Flipped) from radiative $B \rightarrow X_{s} \gamma$ decays [21, 22]; from $H / A \rightarrow \tau \tau$ LHC searches [23, 24] a bound (for Type II) rising approximately linearly from $m_{22} \gtrsim 300 \mathrm{GeV}$ at $\tan \beta=10$ to $m_{22} \gtrsim 1000 \mathrm{GeV}$ at $\tan \beta=60$, and; early Landau poles (for Type II, Flipped, and LS models) when $\tan \beta \gtrsim 70$ [25].

\subsection{Neutrino masses and leptogenesis}

The neutrino mass matrix is given by

$$
m_{v}=\frac{v_{2}^{2}}{2} y_{v} \mathscr{D}_{M}^{-1} y_{v}^{T} \approx \frac{1}{\tan ^{2} \beta}\left(\frac{v^{2}}{2} y_{v} \mathscr{D}_{M}^{-1} y_{v}^{T}\right),
$$

suppressed with respect to the standard seesaw Eq. 1.2. Clearly a smaller $v_{2}$ forces a larger $y_{v}$ in order to realise the observed neutrino masses, and it is the size of the $y_{v}$ entries which control the

\footnotetext{
${ }^{2}$ The relevant quantity is $\cos (\alpha-\beta) \sim \frac{1}{\tan \beta} \frac{v_{1}^{2}}{m_{22}^{2}} \ll 1$, see Ref. [2].
} 
amount of $C P$ violation present in $N_{1}$ decays. As such, and if leptogenesis proceeds in a sufficiently similar way (we will soon discuss that it does), the Davidson-Ibarra bound Eq. 1.3 is suppressed by $\approx 1 / \tan ^{2} \beta$ and the scale of successful leptogenesis can be lowered. This is illustrated in Fig. 2 .

The observed BAU is produced analogously to standard hierarchical thermal leptogenesis (see e.g. Refs. [26, 27] for reviews), via the out-of-equilibrium, $C P$ violating decays of the lightest righthanded neutrino, but now into the second Higgs doublet: $N_{1} \rightarrow l \Phi_{2}$. When only decays and inverse decays are considered, and in the one-flavour approximation, the decay parameter $K$ characterises the asymmetry:

$$
K=\frac{\Gamma_{D}}{\left.H\right|_{T=M_{1}}}=\frac{\tilde{m}_{1}}{m_{*}}
$$

where $\Gamma_{D}$ is the $N_{1}$ decay rate, $H$ is the expansion rate of the Universe, $\tilde{m}_{1}$ is the effective neutrino mass, and $m_{*}$ is the equilibrium neutrino mass,

$$
\Gamma_{D}=\frac{1}{8 \pi}\left(y_{v}^{\dagger} y_{v}\right)_{11} M_{1}, \quad H \approx \frac{17 T^{2}}{M_{P l}}, \quad \tilde{m}_{1} \equiv \frac{\left(y_{v}^{\dagger} y_{v}\right)_{11} v_{2}^{2}}{2 M_{1}}, \quad m_{*} \approx \frac{1.1 \times 10^{-3} \mathrm{eV}}{\tan ^{2} \beta} .
$$

With these definitions we have the familiar weak and strong washout regimes when $K \ll 1$ and $K \gg 1$, respectively. Note that $m_{*}$ is smaller than its usual value in standard leptogenesis.

The $2 \leftrightarrow 2$ scatterings with $\Delta L=1$ are important for washout and early $N_{1}$ production in the non-thermal weak washout regime. Electroweak scatterings are identical to those in the standard scenario, however those involving top quarks $(N l \leftrightarrow t q, N t \leftrightarrow l q, N q \leftrightarrow l t)$ are absent by construction. Instead, at sufficiently large $\tan \beta$, top scatterings are replaced by the analogous bottom quark and tau lepton scatterings (depending on the $v 2 \mathrm{HDM}$ type). ${ }^{3}$ All of these scatterings are proportional to $\left(y_{v}^{\dagger} y_{v}\right)_{11}$, as are the decays and inverse decays, so that they can only result in a minor departure from the standard scenario.

The $2 \leftrightarrow 2$ scatterings with $\Delta L=2\left(\Phi_{2} l \leftrightarrow \bar{\Phi}_{2} \bar{l}, \Phi_{2} \Phi_{2} \leftrightarrow l l\right)$ can however have a much larger impact. The rate of these scatterings is proportional to $\operatorname{Tr}\left[\left(y_{v} y_{v}^{T}\right)\left(y_{v} y_{v}^{T}\right)^{\dagger}\right]$; comparing this to the rate of decays, inverse decays, and $\Delta L=1$ scatterings, we have

$$
\frac{\operatorname{Tr}\left[\left(y_{v} y_{v}^{T}\right)\left(y_{v} y_{v}^{T}\right)^{\dagger}\right]}{\left(y_{v}^{\dagger} y_{v}\right)_{11}} \propto \frac{M_{N_{1}}^{2} \bar{m}^{2}}{v_{2}^{4}} \frac{v^{2}}{2 M_{N_{1}} K \times 10^{-3} \mathrm{eV}},
$$

where $\bar{m}^{2}=\sum m_{i}^{2} \gtrsim(0.05 \mathrm{eV})^{2}$. Clearly this ratio increases as $v_{2}$ decreases ( $\tan \beta$ increases). For $T \lesssim M_{N_{1}} / 3$ the $\Delta L=2$ scattering rate is approximated (in the one-flavour approximation) by [26]

$$
\frac{\Gamma_{\Delta L=2}}{H} \approx \frac{T}{2.2 \times 10^{13} \mathrm{GeV}}\left(\frac{\bar{m}}{0.05 \mathrm{eV}}\right)^{2}\left(\frac{v}{v_{2}}\right)^{4},
$$

In Fig. 2 we show two regions of interest for these scatterings: when they are in equilibrium at $T \lesssim M_{N_{1}} / 3$ and $T \sim 100 \mathrm{GeV}$. In these regions strong $\Delta L=2$ washout can potentially destroy any asymmetry created. ${ }^{4}$

Lastly we note that, since natural leptogenesis will generically be occurring at $T<10^{9} \mathrm{GeV}$, flavour effects cannot be ignored (see e.g. Refs. [28, 29, 30, 31]). It is known, for example, that

\footnotetext{
${ }^{3} \mathrm{~A}$ large $y_{\tau}$ also introduces new scattering diagrams: $N \Phi_{2} \leftrightarrow \tau \Phi_{2}, \tau N \leftrightarrow \Phi_{2} \bar{\Phi}_{2}$.

${ }^{4}$ Note that these regions are not applicable in the non-perturbative regime indicated by the grey dotted lines in Fig. 2.
} 



Figure 2: Left: Bounds on the $v 2 \mathrm{HDM}$ as a function of $v_{2}$. Shown (as labelled) are the Davidson-Ibarra bound, the Vissani naturalness bound, and the areas of parameter space with strong $\Delta L=2$ scattering washout. The grey dotted lines indicate the $v_{2}$ below which the Yukawas hit a Landau pole before $M_{N_{1}}$ in the Type II, Flipped, and LS v2HDMs right-to-left. Right: As in Left but for the Ma model. Each bound is evaluated at $m_{22}=500 \mathrm{GeV}$. (Figure adapted from Ref. [2]).

flavour alignments can protect the asymmetry from washout [14]. It is therefore plausible that successful leptogenesis is still possible in the strong $\Delta L=2$ washout region. These effects deserve further study. Still, the overall picture should not dramatically change, and the (rescaled) DavidsonIbarra bound is expected to hold (as it does the standard case with flavour effects [31, 32]).

\subsection{Naturalness}

There are three explicit scales in the $v 2 \mathrm{HDM}: m_{11}^{2}, m_{22}^{2}$, and the $M_{j}$. A natural scenario is achieved if (1) $m_{11}^{2}$ is protected from $m_{22}^{2}$ corrections, and (2) $m_{22}^{2}$ is protected from $M_{j}$ corrections. We consider each in turn.

In the typical situation where $m_{12}^{2} \ll\left|m_{11}^{2}\right|, m_{11}^{2}$ sets the Higgs mass $\left(m_{h}^{2} \approx-2 m_{11}^{2}\right)$ so that $m_{11}^{2} \approx-(88 \mathrm{GeV})^{2}$. Naturalness considerations will imply, because both $\Phi_{i}$ have gauge charges, the $m_{22}^{2}$ scale cannot be very much separated from $m_{11}^{2}$. At one-loop, the $m_{11}^{2}$ RGE is

$$
\frac{d m_{11}^{2}}{d \ln \mu_{R}}=\frac{1}{(4 \pi)^{2}}\left[\left(4 \lambda_{3}+2 \lambda_{4}\right) m_{22}^{2}+\mathscr{O}\left(m_{11}^{2}\right)\right]
$$

It appears that the limit $\lambda_{3,4} \rightarrow 0$ protects $m_{11}^{2}$ from $m_{22}^{2}$, however these couplings are reintroduced by gauge loops:

$$
\frac{d \lambda_{3}}{d \ln \mu_{R}}=\frac{1}{(4 \pi)^{2}}\left[\frac{3}{4}\left(g_{Y}^{4}-2 g_{Y}^{2} g_{2}^{2}+3 g_{2}^{4}\right)+\ldots\right], \quad \frac{d \lambda_{4}}{d \ln \mu_{R}}=\frac{1}{(4 \pi)^{2}}\left[3 g_{Y}^{2} g_{2}^{2}+\ldots\right] .
$$

This is another way of saying there exists an irremovable pure-gauge two-loop correction to $m_{11}^{2}$ that is proportional to $m_{22}^{2}$ (see Ref. [33] for the two-loop result). Bounding directly just the two-loop pure-gauge contribution by $1 \mathrm{TeV}^{2}$ results in a conservative naturalness bound of $m_{22} \lesssim$ $10^{5} \mathrm{GeV}$. It is also illuminating to consider the condition $\Delta\left(M_{P l}\right)<10$; in Ref. [2] we showed that 
this implies a stringent naturalness bound $m_{22} \lesssim$ few $\times 10^{3} \mathrm{GeV}$. These bounds are not to be taken too seriously, they are merely sufficient to demonstrate that a $\mathrm{TeV}$-scale $m_{22}$ is not only experimentally allowed in all $v 2 \mathrm{HDM}$ Types, but can also remain natural.

Bounding the $m_{22}^{2}$ RGE directly by $d m_{22}^{2} / d \ln \mu_{R}<1 \mathrm{TeV}^{2}$ results in naturalness bounds on the $M_{j}$ given by Eq. 2.3 with the replacement $v \rightarrow v_{2} .{ }^{5}$ This bound is depicted in Fig. 2. A similar bound is obtained for $m_{22} \sim 1 \mathrm{TeV}$ and a fine-tuning criterion $\Delta\left(M_{P l}\right)<10$. We can now read off the region of parameter space of interest for natural leptogenesis: we find, depending on the $v 2 \mathrm{HDM}$ Type, fully perturbative solutions with $0.3 \lesssim v_{2} / \mathrm{GeV} \lesssim 30$ and $10^{3} \lesssim M_{N_{1}} / \mathrm{GeV} \lesssim 10^{7}$.

\subsection{The Ma model}

Lastly let us comment that our discussion extends analogously to the Ma model of radiative neutrino mass [34]. In this model the $2 \mathrm{HDM}$ potential is given by Eq. 3.2 with $m_{12}^{2}=0$ and an additional explicit $U(1)$ breaking term $\frac{\lambda_{5}}{2}\left[\left(\Phi_{1}^{\dagger} \Phi_{2}\right)^{2}+\left(\Phi_{2}^{\dagger} \Phi_{1}\right)^{2}\right]$, retaining a $Z_{2}$ symmetry which remains unbroken $\left(v_{2}=0\right)$. For $M_{N}^{2} \gg m_{22}^{2}, v^{2}$, the radiatively generated neutrino mass matrix is

$$
\left(m_{v}\right)_{i j} \approx \frac{v^{2}}{2} \frac{\left(y_{v}\right)_{i k}\left(y_{v}^{T}\right)_{k j}}{M_{k}} \frac{\lambda_{5}}{8 \pi^{2}}\left(\ln \left[\frac{2 M_{k}^{2}}{\left(m_{H}^{2}+m_{A}^{2}\right)}\right]-1\right) .
$$

Arguments analogous to those already presented, ${ }^{6}$ but with $v_{2}^{2} \rightarrow v^{2} \frac{\lambda_{5}}{8 \pi^{2}}\left(\ln \left[2 M_{N_{1}}^{2} /\left(m_{H}^{2}+m_{A}^{2}\right)\right]-1\right)$ (c.f. Eqs. 3.11 and 3.4) lead to a rescaling of the Davidson-Ibarra bound, the Vissani bound, and the strong $\Delta L=2$ scattering regions. These bounds are shown in Fig. 2 for an example mass $m_{22}=500 \mathrm{GeV}$ (but they are only mildly dependent on $m_{22}$ ). The region $10^{-5} \lesssim \lambda_{5} \lesssim 10^{-1}$ with $10^{3} \lesssim M_{N_{1}} / \mathrm{GeV} \lesssim 10^{7}$ can naturally realise neutrino masses and hierarchical leptogenesis. As well, $H$ or $A$ is a viable dark matter candidate.

\section{Conclusion}

The three-flavour Type I seesaw model is a simple way to explain neutrino masses and the BAU via hierarchical thermal leptogenesis. However, as we proved in Sec. 2, it cannot do so without introducing a naturalness problem [1]. In Sec. 3 we listed some minimal ways to adapt the model to avoid this inconsistency: dominiant initial $N_{1}$ abundancy; resonant leptogenesis; neutrino oscillations; introducing an independent source of $C P$ violation in $N_{1}$ decays; partial loop cancellations; supersymmetry, and; reducing the (possibly effective) vev entering the seesaw. We showed how to construct viable, natural v2HDMs which utilise the latter mechanism [2]. Such models predict an automatically SM-like Higgs boson, (maximally) TeV-scale scalar states, and low- to intermediate-scale hierarchical leptogenesis with $10^{3} \mathrm{GeV} \lesssim M_{N_{1}} \lesssim 10^{7} \mathrm{GeV}$. One version (the radiative Ma model) also includes a dark matter candidate.

\section{Acknowledgments}

This work was supported in part by the Australian Research Council. Figures are reproduced from [1, 2]. I would like to thank the conference organisers for their hospitality.

\footnotetext{
${ }^{5}$ Actually, the Vissani bound may be relaxed since $m_{22}$ can be naturally TeV-scale. For clarity we will not consider this here; see our Ref. [2] for more details.

${ }^{6}$ See Ref. [2] and Appendices therein for some minor caveats
} 


\section{References}

[1] J. D. Clarke, R. Foot, and R. R. Volkas, Electroweak naturalness in the three-flavor type I seesaw model and implications for leptogenesis, Phys. Rev. D91 (2015), no. 7 073009, [arXiv:1502.01352].

[2] J. D. Clarke, R. Foot, and R. R. Volkas, Natural leptogenesis and neutrino masses with two Higgs doublets, Phys. Rev. D92 (2015), no. 3 033006, [arXiv: 1505.05744 ].

[3] P. Minkowski, $\mu \rightarrow$ er at a Rate of One Out of 1-Billion Muon Decays?, Phys.Lett. B67 (1977) 421.

[4] R. Mohapatra and G. Senjanovic, Neutrino Mass and Spontaneous Parity Violation, Phys.Rev.Lett. 44 (1980) 912.

[5] T. Yanagida, Horizontal symmetry and masses of neutrinos, Conf.Proc. C7902131 (1979) 95-99.

[6] M. Gell-Mann, P. Ramond, and R. Slansky, Complex Spinors and Unified Theories, Conf.Proc. C790927 (1979) 315-321, [arXiv:1306.4669].

[7] M. Fukugita and T. Yanagida, Baryogenesis Without Grand Unification, Phys.Lett. B174 (1986) 45.

[8] S. Davidson and A. Ibarra, A Lower bound on the right-handed neutrino mass from leptogenesis, Phys.Lett. B535 (2002) 25-32, [hep-ph/ 0202239 ].

[9] G. Giudice, A. Notari, M. Raidal, A. Riotto, and A. Strumia, Towards a complete theory of thermal leptogenesis in the SM and MSSM, Nucl.Phys. B685 (2004) 89-149, [hep-ph/ 0310123$].$

[10] F. Vissani, Do experiments suggest a hierarchy problem?, Phys.Rev. D57 (1998) 7027-7030, [hep-ph/9709409].

[11] J. Ellis, K. Enqvist, D. Nanopoulos, and F. Zwirner, Observables in Low-Energy Superstring Models, Mod.Phys.Lett. A1 (1986) 57.

[12] R. Barbieri and G. Giudice, Upper Bounds on Supersymmetric Particle Masses, Nucl.Phys. B306 (1988) 63.

[13] P. Di Bari, Seesaw geometry and leptogenesis, Nucl.Phys. B727 (2005) 318-354, [hep-ph/0502082].

[14] O. Vives, Flavor dependence of CP asymmetries and thermal leptogenesis with strong right-handed neutrino mass hierarchy, Phys.Rev. D73 (2006) 073006, [hep-ph / 0512160 ].

[15] G. Engelhard, Y. Grossman, E. Nardi, and Y. Nir, The Importance of N2 leptogenesis, Phys.Rev.Lett. 99 (2007) 081802, [hep-ph/ 0612187$].$

[16] A. Pilaftsis and T. Underwood, Resonant leptogenesis, Nucl.Phys. B692 (2004) 303-345, [hep-ph/0309342].

[17] E. Akhmedov, V. Rubakov, and A. Smirnov, Baryogenesis via neutrino oscillations, Phys.Rev.Lett. 81 (1998) 1359-1362, [hep-ph/9803255].

[18] F. Bazzocchi and M. Fabbrichesi, Little hierarchy problem for new physics just beyond the LHC, Phys.Rev. D87 (2013), no. 3 036001, [arXiv:1212.5065].

[19] M. Fabbrichesi and A. Urbano, Naturalness redux: The case of the neutrino seesaw mechanism, Phys. Rev. D92 (2015) 015028, [arXiv: 1504.05403 ].

[20] LEP Higgs Working Group for Higgs boson searches, ALEPH, DELPHI, L3, OPAL Collaboration, T. Junk et al., Search for charged Higgs bosons: Preliminary combined results using LEP data collected at energies up to 209-GeV, hep-ex/0107031. 
[21] Heavy Flavor Averaging Group (HFAG) Collaboration, Y. Amhis et al., Averages of b-hadron, c-hadron, and $\tau$-lepton properties as of summer 2014, arXiv: 1412.7515.

[22] M. Misiak et al., Updated NNLO QCD predictions for the weak radiative B-meson decays, arXiv:1503.01789.

[23] ATLAS Collaboration, G. Aad et al., Search for neutral Higgs bosons of the minimal supersymmetric standard model in pp collisions at $\sqrt{s}=8 \mathrm{TeV}$ with the ATLAS detector, JHEP 1411 (2014) 056, [arXiv:1409.6064].

[24] CMS Collaboration, V. Khachatryan et al., Search for neutral MSSM Higgs bosons decaying to a pair of tau leptons in pp collisions, JHEP 1410 (2014) 160, [arXiv: 1408 . 3316 ].

[25] J. Bijnens, J. Lu, and J. Rathsman, Constraining General Two Higgs Doublet Models by the Evolution of Yukawa Couplings, JHEP 1205 (2012) 118, [arXiv: 1111.5760 ].

[26] W. Buchmuller, P. Di Bari, and M. Plumacher, Leptogenesis for pedestrians, Annals Phys. 315 (2005) 305-351, [hep-ph/0401240].

[27] S. Davidson, E. Nardi, and Y. Nir, Leptogenesis, Phys.Rept. 466 (2008) 105-177, [arXiv:0802.2962].

[28] A. Abada, S. Davidson, F.-X. Josse-Michaux, M. Losada, and A. Riotto, Flavor issues in leptogenesis, JCAP 0604 (2006) 004, [hep-ph / 0601083 ].

[29] E. Nardi, Y. Nir, E. Roulet, and J. Racker, The Importance of flavor in leptogenesis, JHEP 01 (2006) 164, [hep-ph/0601084].

[30] A. Abada, S. Davidson, A. Ibarra, F. X. Josse-Michaux, M. Losada, and A. Riotto, Flavour Matters in Leptogenesis, JHEP 09 (2006) 010, [hep-ph / 0605281 ].

[31] S. Blanchet and P. Di Bari, Flavor effects on leptogenesis predictions, JCAP 0703 (2007) 018, [hep-ph/0607330].

[32] F. Josse-Michaux and A. Abada, Study of flavour dependencies in leptogenesis, JCAP 0710 (2007) 009, [hep-ph/0703084].

[33] D. Chowdhury and O. Eberhardt, Global fits of the two-loop renormalized Two-Higgs-Doublet model with soft $Z_{2}$ breaking, arXiv:1503.08216.

[34] E. Ma, Verifiable radiative seesaw mechanism of neutrino mass and dark matter, Phys.Rev. D73 (2006) 077301, [hep-ph/o 601225 ]. 